\title{
$\beta$-Adrenergic receptor blockade arrests myocyte damage and preserves cardiac function in the transgenic $\mathbf{G}_{\mathrm{s} \alpha}$ mouse
}

\author{
Kuniya Asai, ${ }_{1}^{1}$ Gui-Ping Yang, ${ }^{1}$ Yong-Jian Geng, ${ }^{1}$ Gen Takagi,,${ }^{1}$ Sanford Bishop, ${ }^{1}$ \\ Yoshihiro Ishikawa, ${ }^{1}$ Richard P. Shannon, ${ }^{2}$ Thomas E. Wagner, ${ }^{3}$ Dorothy E. Vatner, ${ }^{1}$ \\ Charles J. Homcy, ${ }^{4}$ and Stephen F. Vatner ${ }^{1}$ \\ ${ }^{1}$ Weis Center for Research, Penn State College of Medicine, Danville, Pennsylvania 17822, USA \\ ${ }^{2}$ Cardiovascular and Pulmonary Research Institute, Allegheny University of the Health Sciences, Pittsburgh, \\ Pennsylvania 15212, USA \\ ${ }^{3}$ Department of Molecular Medicine, Clemson University and the Greenville Hospital System/Clemson University Biomedical \\ Cooperative, Greenville, South Carolina 29605, USA \\ ${ }^{4}$ COR Therapeutics Inc., South San Francisco, California 94080, USA
}

Address correspondence to: Stephen F. Vatner, Weis Center for Research, Penn State College of Medicine, 100 North Academy Avenue, Danville, Pennsylvania 17822, USA. Phone: (570) 271-6659; Fax: (570) 271-6701; E-mail: svatner@psghs.edu.

Received for publication May 25, 1999, and accepted in revised form July 27, 1999.

Transgenic (TG) mice with cardiac $\mathrm{G}_{\mathrm{s} \alpha}$ overexpression exhibit enhanced inotropic and chronotropic responses to sympathetic stimulation, but develop cardiomyopathy with age. We tested the hypothesis that cardiomyopathy in TG mice with $\mathrm{G}_{\mathrm{s} \alpha}$ overexpression could be averted with chronic $\beta$-adrenergic receptor ( $\beta$-AR) blockade. TG mice and age-matched wild-type littermates were treated with the $\beta$-AR blocker propranolol for 6-7 months, starting at a time when the cardiomyopathy was developing but was not yet severe enough to induce significant cardiac depression (9.5 months of age), and ending at a time when cardiac depression and cardiomyopathy would have been clearly manifest (16 months of age). Propranolol treatment, which can induce cardiac depression in the normal heart, actually prevented cardiac dilation and the depressed left ventricular function characteristic of older TG mice, and abolished premature mortality. Propranolol also prevented the increase in myocyte cross-sectional area and myocardial fibrosis. Myocyte apoptosis, already apparent in 9-month-old TG mice, was actually eliminated by chronic propranolol. This study indicates that chronic sympathetic stimulation over an extended period is deleterious and results in cardiomyopathy. Conversely, $\beta$ AR blockade is salutary in this situation and can prevent the development of cardiomyopathy.

J. Clin. Invest. 104:551-558 (1999).

\section{Introduction}

Despite intense investigation, mortality due to heart failure remains preeminent in the United States, largely because the etiologic processes underlying this disease remain enigmatic. In part, this is due to a complex dialectical process that is generally initiated by acute cardiac injury or overload, and followed by a series of compensatory adjustments and subsequent failure to maintain compensation, resulting in cardiac dilation and heart failure or sudden death. Compensatory adjustments include neural/hormonal changes and myocardial hypertrophy. Although both of these broad types of compensatory mechanisms are initially helpful, they do not prevent decompensation and heart failure. In fact, there is one point of view that holds that these compensatory mechanisms actually may facilitate decompensation. For example, the hypertrophic response, per se, has secondary effects that ultimately can be deleterious, e.g., the accompanying impairment of subendocardial coronary reserve in the hypertrophied heart (1). Similarly, when the heart is stressed, as occurs in the pathogenesis of heart failure, a variety of autocrine, paracrine, neural, and hormonal pathways are triggered and activated tonically, which can also affect the heart adversely over the long term. Cardiologists and physiologists have wrestled with the central question of whether these adaptive processes can be modulated in such a manner that the maladaptive responses can be avoided.

In an attempt to delineate signals and pathways that impact the heart under situations of stress, studies of genetically manipulated mice have been undertaken by either enhancing or alternatively deleting the activity of a signaling pathway in the heart (2-14). Our laboratory has focused on the cardiac $\beta$-adrenergic receptor- $\mathrm{G}_{\mathrm{s}}$-adenylyl ( $\beta-A R-\mathrm{G}_{\mathrm{s}}$-adenylyl) cyclase pathway, which, when activated by norepinephrine release from sympathetic nerve terminals, delivers a potent physiological stimulus to enhance an increase both in heart rate and in contractile force. Several years ago, we genetically enhanced the postsynaptic $\beta-A R-G_{s}-$ adenylyl cyclase pathway in cardiocytes by overexpressing $\mathrm{G}_{\mathrm{s} \alpha}$, the stimulatory $\mathrm{G}$ protein $\alpha$ subunit, in the hearts of transgenic (TG) mice (8). In short, over their lifetime, they exhibit marked hyperresponsiveness to catecholamine stimulation, as exhibited by 


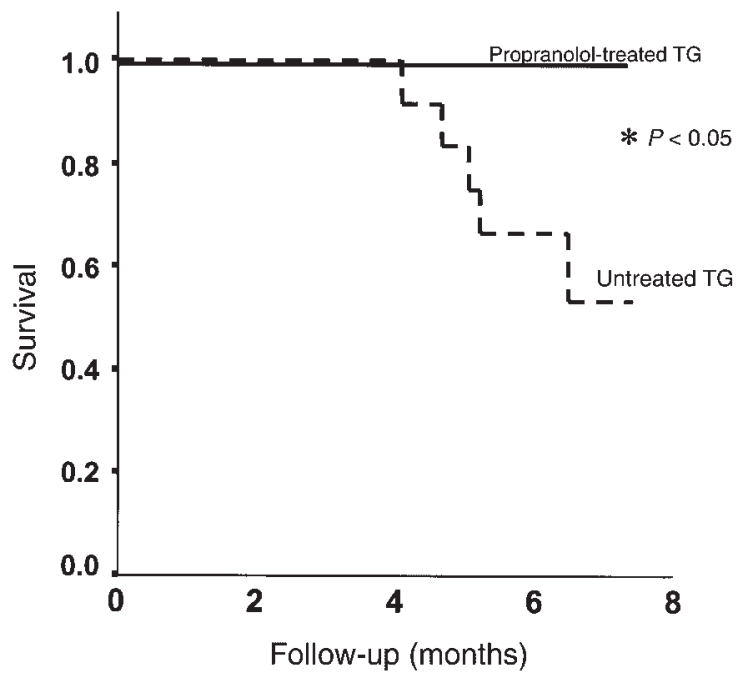

Figure 1

Kaplan-Meier analysis of survival curves of propranolol-treated and untreated TG mice. Propranolol treatment significantly $(P<0.05$ by log-rank test) reduced mortality in TG mice.

supranormal ejection fractions and a marked increase in heart rate (9-11); interestingly, though, they lack a typical desensitization response (12). As these animals age, there is increasing myocyte cytoplasmic and nuclear degeneration and cell loss, increased positive terminal deoxynucleotidyl transferase-mediated dUTP nick end-labeling (TUNEL) staining characteristic of apoptosis (13), myocardial interstitial and replacement fibrosis, myocyte hypertrophy, cardiac dilation, depressed left ventricular (LV) function, arrhythmias, and sudden death - a picture reminiscent of human cardiomyopathy $(9,14)$. Unlike other TG models, this pattern evolves well beyond the period of normal growth and development. Thus, this model recapitulates the pattern of tonic and chronic sympathetic stimulation to the heart that is characteristic of heart failure. But, unlike the disease process, $\beta$-AR overactivity appears to be the inciting stimulus, as opposed to a compensating adjustment to an initiating insult of cardiac malfunction; accordingly, $\beta$-AR overactivity becomes useful in understanding mechanisms of heart failure related to the sympathetic nervous system. We now report on the long-term effects of blocking this pathway with a $\beta$ AR antagonist to determine whether the cardiac damage attributed to the persistent stimulation of the $\beta$ AR signaling pathway in this model can, in fact, be prevented or reversed.

\section{Methods}

Animals. Twenty-one TG mice $(9.4 \pm 0.1$ months old) and 24 age-matched wild-type (WT) littermates (9.5 \pm 0.1 months old) of either sex from the same genetic background as the TG mice were studied. Briefly, the transgene consists of a rat $\alpha$-myosin heavy chain promoter linked to a $G_{s \alpha}$ minigene coding for the short isoform of $\mathrm{G}_{\mathrm{s} \alpha}$ (8). Animals used in the present study were maintained in accordance with guidelines from the National Institutes of Health (15).

Chronic treatment with $\beta$-AR blockade. TG and WT mice were randomly assigned to either a propranololtreated group (TG, $n=9$; WT, $n=14$ ) or an untreated group (TG, $n=12$; WT, $n=10$ ). Propranolol, which is a nonselective $\beta$-AR blocker, was administered in the drinking water at a concentration of $0.5 \mathrm{~g} / \mathrm{L}$, or tap water was given to the untreated group. In preliminary experiments, we determined that propranolol blocked the inotropic response to isoproterenol infusion in TG mice at this concentration; i.e., the increase in LV ejection fraction (EF) normally observed with isoproterenol $(0.02 \mu \mathrm{g} / \mathrm{kg}$ per minute) did not occur in the propranolol-treated mice. Water with freshly added drug was changed 3 times weekly, and mice were treated for 6-7 months.

Echocardiography. Echocardiographic studies were performed in 19 TG mice (9 propranolol-treated and 10 untreated, $14.4 \pm 0.1$ months old) and 24 WT controls (14 propranolol-treated and 10 untreated, 14.8 \pm 0.2 months old). In addition, we also examined echocardiographic studies in 10 younger TG mice (8.8 \pm 0.1 months old). After determination of body weight, mice were anesthetized with ketamine $(0.065$ $\mathrm{mg} / \mathrm{g})$, acepromazine $(0.002 \mathrm{mg} / \mathrm{g})$, and xylazine $(0.013 \mathrm{mg} / \mathrm{g})$ injected intraperitoneally. The procedure for echocardiography has been described in previous reports from our laboratory $(9,14)$. In brief, electrocardiographic leads were attached to each limb using needle electrodes (Grass Instrument Co., Quincy, Massachusetts, USA). Echocardiography was performed using ultrasonography (Apogee X-200; Interspec Inc., Amber, Pennsylvania, USA). A dynamically focused 9-MHz annular array transducer was applied from below, using a warmed saline bag as a standoff. M-mode measurements of LV internal diameter (LVID) were made from more than 3 beats and averaged. End-diastolic measurements were taken at the time of the apparent maximal LV diastolic dimension. End-systolic measurements were made at the time of the most anterior systolic excursion of the posterior wall. LVEF was calculated by the cubed method as follows: $\mathrm{LVEF}=\left[\left(\mathrm{LVIDd}^{3}-\left(\mathrm{LVIDs}^{3}\right] /(\mathrm{LVIDd})^{3}\right.\right.$, where d indicates diastolic and $\mathrm{s}$ indicates systolic. Using aortic ejection time (ET) obtained from the Doppler tracing of the LV outflow tract, we also calculated the mean velocity of circumferential fiber shortening corrected for heart rate (Vcfc; in s ${ }^{-1 / 2}$ ) as follows: [(LVIDd - LVIDs)/LVIDd]/[ET/R-R interval ${ }^{1 / 2}$ (in seconds)].

Heart rate. Heart rate was measured using telemetry techniques in the conscious, unrestrained state in 16 TG mice ( 9 propranolol-treated and 7 untreated, 15.6 \pm 0.1 months old) and $23 \mathrm{WT}$ mice (14 propranololtreated and 9 untreated, $15.9 \pm 0.1$ months old). Mice were anesthetized as already described, and a telemetry transducer (TA10EA-F20; Data Science Co., St. Paul, Minnesota, USA) was implanted in the peritoneal cav- 
ity with paired electrodes placed subcutaneously over the thorax (chest bipolar electrocardiogram leads) (10). Experiments were initiated 3-5 days after recovery from surgical instrumentation. Mice with implanted telemetry devices were housed in individual cages with free access to food and water, and were exposed to 12 -hour light/ 12-hour dark cycles.

Arterial pressure. Because 1 propranolol-treated TG mouse died after anesthesia, arterial pressure was measured in 15 TG mice (8 propranolol-treated and 7 untreated, $16.1 \pm 0.1$ months old) and $23 \mathrm{WT}$ mice (14 propranolol-treated and 9 untreated, $16.3 \pm 0.1$ months old). The mice were anesthetized as already described, and arterial blood pressures were measured by a $1.4 \mathrm{~F}$ micromanometer catheter (Millar Instruments Inc., Houston, Texas, USA), which was inserted into the carotid artery.

Histology. Histological studies were conducted in hearts from 21 older TG mice $(15.7 \pm 0.2$ months old), 23 older WT mice $(16.3 \pm 0.1$ months old), and 10 untreated younger TG mice ( $8.9 \pm 0.1$ months old). The heart was perfused with a brief saline wash followed by paraformaldehyde or formalin via a 21-gauge needle inserted directly into the LV apex. All hearts were fixed by perfusion fixation with $3 \%$ paraformaldehyde, except for the hearts of 5 untreated TG mice that died prematurely, and were fixed with $10 \%$ buffered formalin. All animals were anesthetized as already described. The heart was dissected to remove the atria and right ventricular free wall, and each portion was weighed. Fixed tissues were dehydrated, embedded in paraffin, sectioned at $6-\mu \mathrm{m}$ thickness, and stained with hematoxylin and eosin, Gomori's aldehyde fuchsin trichrome, and picric acid/sirius red. Heart sections were also dehydrated and embedded in glycol methacrylate, sectioned at $1-\mu \mathrm{m}$ thickness, and stained with methylene bluebasic fuchsin for light-microscopic examination. Methacrylate sections were also stained with silver-gold (Accustain; Sigma Diagnostics, St. Louis, Missouri, USA) for basement membrane to outline cardiac myocytes for cross-sectional area measurement.

Myocardial connective tissue was quantitatively analyzed on a cross-section of LV obtained mid-distance from base to apex and stained with picric acid/sirius red. Images were obtained from a Sony CCD video camera (Sony Electronics Inc., Park Ridge, New Jersey, USA) attached to a Nikon E800 microscope (Nikon Inc. Melville, New York, USA) with a $\times 60$ objective, and were analyzed with MetaMorph image analysis software (Universal Imaging Corp., West Chester, Pennsylvania, USA).

Myocyte cross-sectional area was measured from images captured from silver-stained $1-\mu \mathrm{m}$-thick methacrylate sections obtained mid-distance from base to apex. Suitable cross-sections were defined as having nearly circular capillary profiles and circular-to-oval myocyte sections. No correction for oblique sectioning was made. The outline of 100-200 myocytes was traced in the LV of each animal, using the MetaMorph image
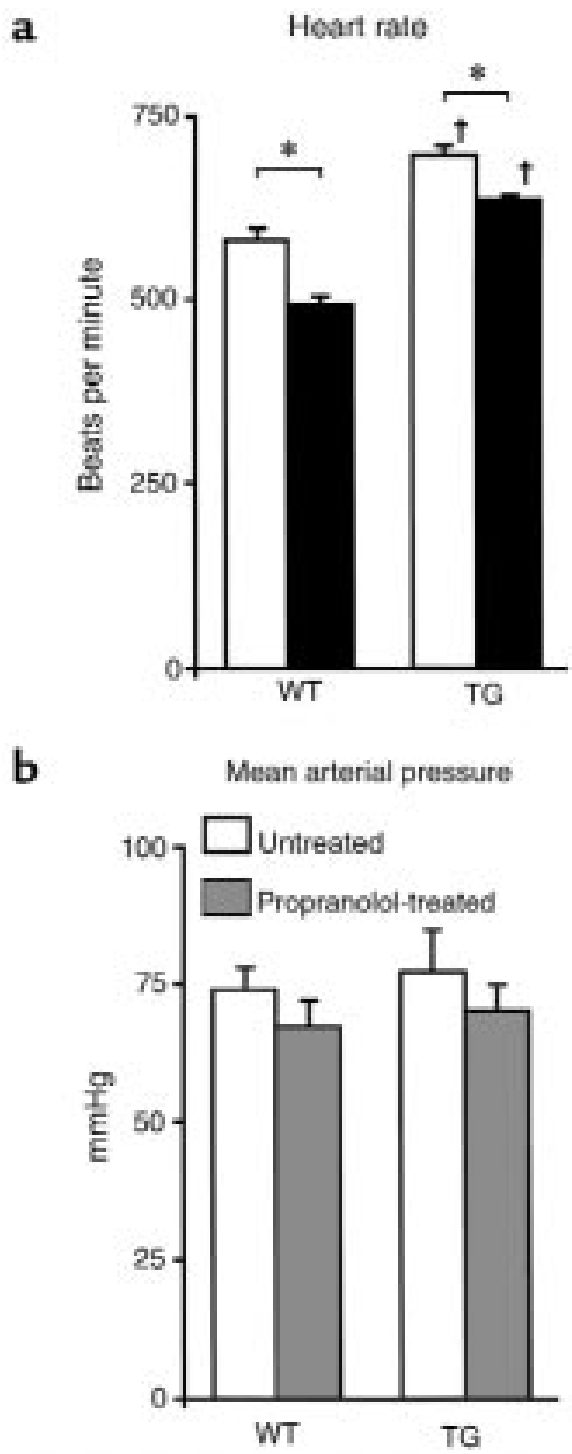

\section{Figure 2}

The effects of chronic propranolol treatment on heart rate in conscious mice (a) and on arterial blood pressure in anesthetized mice (b). Conscious heart rate, derived using a telemetry system, was elevated $(P<0.05)$ in untreated 16-month-old TG mice compared with untreated 16-month-old WT mice. Chronic propranolol treatment reduced conscious heart rate significantly $(P<0.05)$ in both 16 month-old TG and 16-month-old WT mice. Mean arterial pressure also tended to decrease in the propranolol-treated groups, but did not reach significance. ${ }^{*} P<0.05$, untreated vs. propranolol-treated mice. ${ }^{\dagger} P<0.05$, TG mice vs. corresponding WT mice.

system software to determine myocyte cross-sectional area. The mean area was calculated for the LV in each animal, and the group mean was calculated for each region and group.

DNA fragmentation was detected in situ by using TUNEL on paraffin sections of the mouse hearts from TG and WT mice, as we have done previously (13). After the TUNEL procedure, the slides were washed in PBS, mounted in a Vector DAPI medium, and observed under a fluorescence microscope. The 

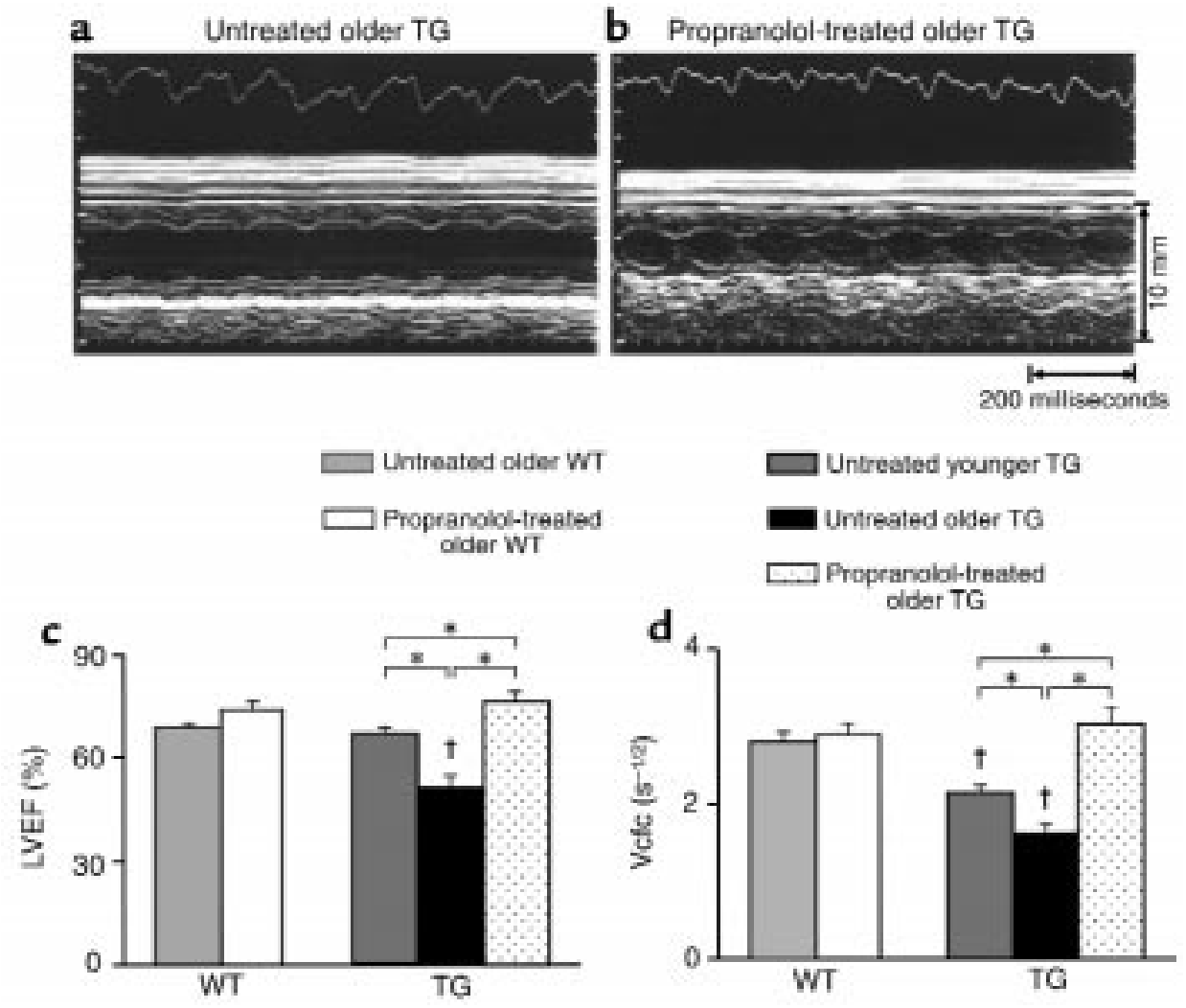

Figure 3

LV function assessed by echocardiography. Shown are representative LV M-mode echocardiographic recordings (a and $\mathbf{b})$ and average data for LV function ( $\mathbf{c}$ and $\mathbf{d}$ ) in both untreated and propranolol-treated older TG mice at 15 months. Propranolol prevented LV dilation in older TG mice. LVEF and mean Vcfc are compared in untreated older (14- to 15-month-old) WT (light gray bars; $n=10$ ), propranolol-treated WT (open bars; $n=14$ ), untreated younger (9-month-old) TG (dark gray bars; $n=10$ ), untreated older TG (black bars; $n=10)$, and propranolol-treated older TG mice (dotted bars; $n=9)$. LV function was significantly $(P<0.05)$ depressed in untreated older TG mice compared with age-matched untreated older WT mice, and depressed LV function was already observed in younger TG mice, whose ages are matched to the age of animals when the $\beta$-AR blockade therapy was begun. LV contractile function in propranolol-treated older TG mice was improved compared with that in both untreated younger and older TG mice. Error bars are \pm SEM. ${ }^{*} P<0.05,{ }^{\dagger} P<0.05$ vs. untreated WT mice.

mean number of myocyte nuclei per $\times 40$ field in $\mathrm{LV}$ regions was determined by manual counting of DAPIstained nuclei with ultraviolet excitation. At the same magnification, a minimum of 20 fields in the $\mathrm{LV}$ regions of each heart were examined for TUNEL-positive myocytes. All morphometric measurements were performed by at least 2 independent individuals in a blinded manner.

Statistics. All data were reported as mean \pm SEM. Multiple group comparisons were analyzed by 1 -way ANOVA, followed by post hoc comparisons between groups using the least significant difference test or unpaired Student's $t$ test. The $\chi^{2}$ test was used to compare differences in premature mortality. Survival curves were established using a Kaplan-Meier survival analysis. Comparisons between 2 groups were performed using the log-rank test. A value of $P<0.05$ was taken as the minimal level of significance.

\section{Results}

Mortality. Propranolol treatment abolished premature mortality in TG mice. During the treatment period (mean duration of follow-up was 6.5 months), there were 5 deaths in untreated TG mice (41.7\%), 1 death in untreated WT mice (10.0\%), and no deaths in propranolol-treated TG mice or WT mice. The Kaplan-Meier survival curves between untreated TG mice and propranolol-treated TG mice were significantly different $(P<0.05)$ (Figure 1).

Conscious heart rate and arterial blood pressure. We assessed the effects of chronic propranolol treatment on heart rate in conscious mice and on arterial blood pressure in anesthetized mice. Conscious heart rate, derived using a telemetry system, was elevated $(P<$ $0.05)$ in untreated 16-month-old TG mice (694 \pm 15 beats/min; $n=7)$ compared with untreated 16 -monthold WT mice $(581 \pm 16$ beats/min; $n=9)$. Chronic propranolol treatment reduced conscious heart rate significantly $(P<0.05)$ in both 16 -month-old TG mice ( $635 \pm 8$ beats $/ \mathrm{min} ; n=9)$ and 16 -month-old WT mice (492 \pm 12 beats/min; $n=14$ ) (Figure 2). Mean arterial pressure also tended to decrease in the propranololtreated groups, but it did not reach significance (untreated TG, $77 \pm 8 \mathrm{mmHg}[n=7]$; propranolol-treat- 
ed TG, $70 \pm 5 \mathrm{mmHg}[n=8]$; untreated WT, $74 \pm 4$ $\mathrm{mmHg}[n=9]$; propranolol-treated WT, $67 \pm 5 \mathrm{mmHg}$ $[n=14]$ in the anesthetized state) (Figure 2).

Echocardiography. Echocardiography was performed to assess LV dimensions and function (Figure 3). LV end-diastolic dimensions were significantly $(P<0.01)$ greater in untreated older (14- to 15-month-old) TG mice $(4.55 \pm 0.16 \mathrm{~mm} ; n=10)$ than in propranololtreated older TG mice $(3.76 \pm 0.19 \mathrm{~mm} ; n=9)$. We calculated the LVEF and the mean Vcfc as indices of LV function. Both LVEF and Vcfc were significantly $(P<$ 0.01 ) depressed in untreated older TG mice (52 $\pm 3 \%$ and $1.63 \pm 0.11 \mathrm{~s}^{-1 / 2}$, respectively) compared with untreated older WT mice $\left(69 \pm 2 \%\right.$ and $2.80 \pm 0.12 \mathrm{~s}^{-1 / 2}$, respectively). These data include those from 3 untreated TG mice that died prematurely. Depressed LV function was already observed in younger (9-month-old) TG mice (Vcfc: $2.14 \pm 0.10 \mathrm{~s}^{-1 / 2}$ ), whose ages are matched to the age of animals when the $\beta$-AR blockade therapy was begun. However, LV function was restored to normal in propranolol-treated older TG mice (LVEF: $76 \pm 3 \%$; Vcfc: $3.03 \pm 0.21 \mathrm{~s}^{-1 / 2}$ ) (Figure 3). LV function was similar in propranolol-treated and untreated older WT mice.

Pathology/histology. The total heart and LV/body weight ratios tended to increase in untreated TG mice (5.64 \pm 0.47 and $4.04 \pm 0.41 \mathrm{mg} / \mathrm{g}$, respectively), but did not reach significance. These ratios in propranololtreated TG mice $(4.67 \pm 0.24$ and $3.37 \pm 0.20 \mathrm{mg} / \mathrm{g}$, respectively) were similar to those of untreated WT mice ( $4.60 \pm 0.28$ and $3.49 \pm 0.20 \mathrm{mg} / \mathrm{g}$, respectively).
Lung/body weight ratio also tended to increase $(P=$ $0.06)$ in untreated TG mice $(7.1 \pm 0.7 \mathrm{mg} / \mathrm{g})$ compared with propranolol-treated TG mice $(5.9 \pm 0.3 \mathrm{mg} / \mathrm{g})$ and untreated WT mice $(6.0 \pm 0.3 \mathrm{mg} / \mathrm{g})$.

The cross-sectional area of cardiac myocytes was significantly increased $(P<0.05)$ in untreated TG mice $\left(200 \pm 6 \mu \mathrm{m}^{2}\right)$ compared with untreated WT mice (178 $\left.\pm 7 \mu \mathrm{m}^{2}\right)$ at 9 months of age, and the difference became more marked at 16 months $\left(276 \pm 9 \mu \mathrm{m}^{2}\right)$. In contrast, there was no significant difference in this parameter between 9-month-old TG mice and propranolol-treated 16-month-old TG mice $\left(208 \pm 5 \mu \mathrm{m}^{2}\right)$. Thus, propranolol treatment arrested further myocyte hypertrophy in TG mice (Figure 4, a and b; and Figure 5a).

Quantitative evaluation of myocardial fibrosis revealed a significant increase in volume percent collagen for untreated 16-month-old TG mice (4.8 \pm $0.6 \%$ ) (Figure 4, c and d; and Figure 5b). Moreover, the fibrotic process was already evident at 9 months of age in the TG mice $(1.8 \pm 0.2 \%)$. Although increased fibrosis was also observed in propranolol-treated 16month-old TG mice $(2.6 \pm 0.5 \%)$, the volume percent collagen of propranolol-treated TG mice was not different from younger (9-month-old) TG mice (Figure $5 b)$. This indicates that propranolol treatment arrested the fibrotic process.

In addition, we recently observed that cardiocyte apoptosis occurred in TG hearts from animals 14-18 months old (13). In the current study, we observed that apoptosis was already present by 9 months of age $(0.15 \pm 0.02 \%$ of myocytes). However, propranolol treatment actually
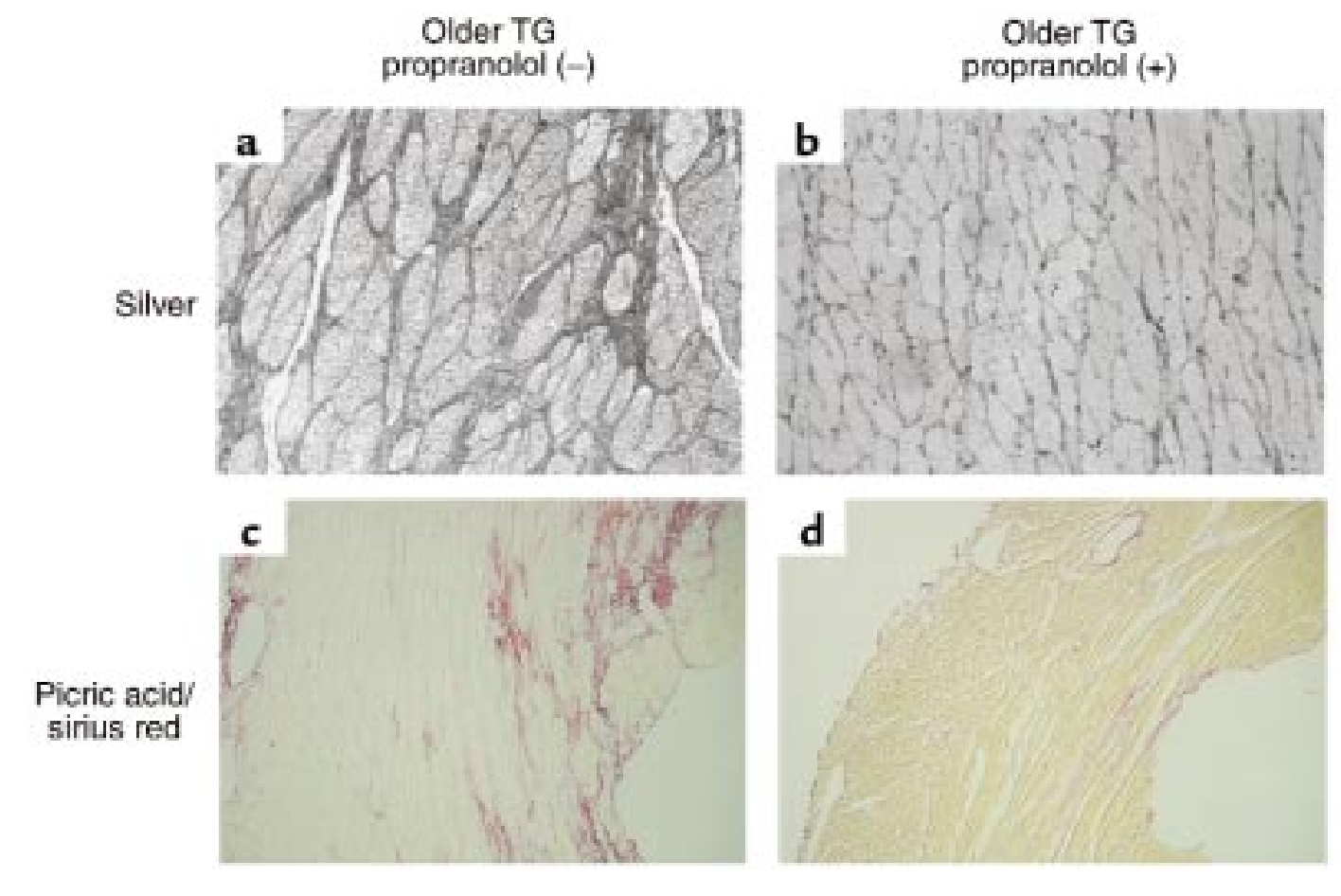

\section{Figure 4}

Morphological analysis in the myocardium of 16-month-old $\mathrm{G}_{\mathrm{s} \alpha} \mathrm{TG}$ mice treated with (b and $\mathbf{d}$ ) and without (a and $\left.\mathbf{c}\right)$ propranolol. (a and b) Silver staining showing myocyte sizes. (c and d) Picric acid/sirius red staining showing collagen content. 


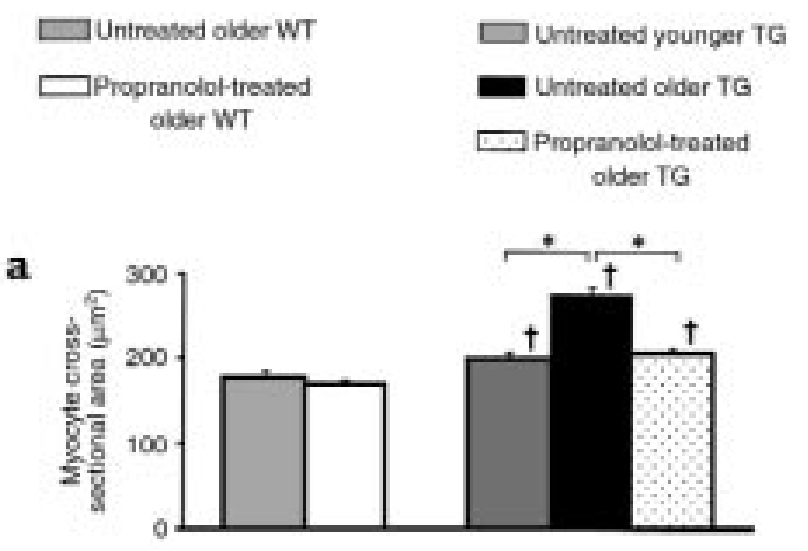

b

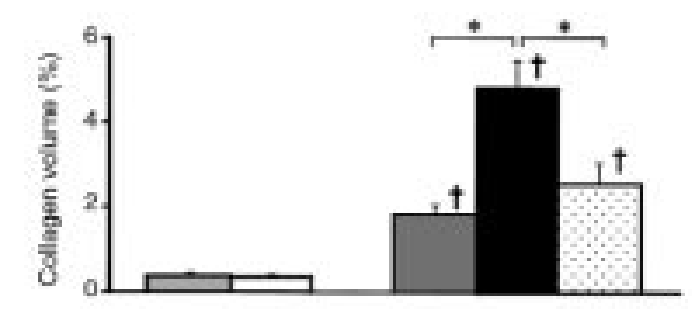

c

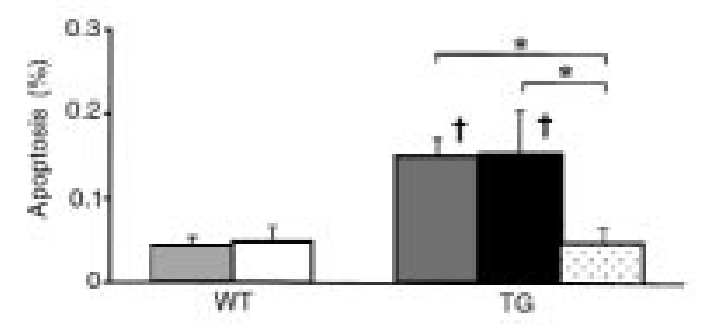

Figure 5

Myocyte cross-sectional area, collagen content, and number of TUNEL-positive myocytes were compared in untreated older (16month-old) WT mice (light gray bars), propranolol-treated older (16-month-old) WT mice (open bars), untreated younger (9-monthold) TG mice (dark gray bars), and propranolol-treated (dotted bars) or untreated (black bars) older (16-month-old) TG mice. (a) Myocyte cross-sectional area measured 100-200 myocytes in each section. (b) Collagen. (c) TUNEL staining. Propranolol prevented the increase in myocyte cross-sectional area and myocyte fibrosis. Furthermore, myocyte apoptosis was eliminated by chronic propranolol. Error bars are \pm SEM. ${ }^{*} P<0.05, \dagger P<0.05$ vs. untreated WT mice.

eliminated the process. There was no significant difference between the number of TUNEL-positive cells in the propranolol-treated 16-month-old TG mice $(0.05 \pm$ $0.02 \%$ of myocytes) and untreated WT mice (0.04 \pm $0.01 \%$ of myocytes), suggesting that the increase in apoptosis over WT mice was not only halted, but actually eliminated, by chronic propranolol therapy (Figure 5c)

The pathology/histology data include those from 5 untreated TG mice that died prematurely. It is also important to note that the data from older WT mice were similar, whether untreated or treated with propranolol.

\section{Discussion}

The heart can call on a variety of mechanisms to deal with the vicissitudes of normal physiological demand.
Sympathetic nerve activation may be most important, as norepinephrine release and reuptake at the synaptic nerve terminal are continually being regulated in a time scale measured in seconds. Thus, the postsynaptic myocardial $\beta$-adrenergic signaling pathway is continuously and rapidly being turned on and off during the course of daily activities. What remains unclear is whether this same pathway, when chronically stimulated (as occurs in heart failure), propagates signals that are ultimately maladaptive or deleterious to the cardiocyte.

The current investigation, using a TG animal model that develops severe cardiomyopathy, provides strong support for the argument that chronic and tonic sympathetic stimulation of the heart is deleterious and that its inhibition can be salutary in the pathogenesis of this disease. One of the unique features of the overexpressed $\mathrm{G}_{\mathrm{s} \alpha}$ model is the impact of the chronicity of the enhanced $\beta$-AR signaling. When the animals are examined as young adults, only enhanced LV function is observed (9-11), as occurs in other models of augmented $\beta$-AR signaling $(2,3)$. However, when the animals reach 9-10 months of age, the cardiomyopathic process has begun, and is in full force by $14-18$ months of age. The older animals are characterized by cardiac dilation, depressed LV function, myocyte fibrosis, hypertrophy, apoptosis, and sudden death $(9,13,14)$. The overexpressed $\mathrm{G}_{\mathrm{s} \alpha}$ mouse model of cardiomyopathy differs from other genetically engineered mouse models of dilated cardiomyopathy (16-23) that develop cardiac dysfunction and cardiomyopathy more rapidly. However, the extent of depressed LV function in untreated TG mice with $\mathrm{G}_{\mathrm{s} \alpha}$ overexpression (22\% fractional shortening) is similar to that observed in other models $(15-25 \%)(16,17,21,22)$. It is important to note that a recent study with overexpression of $\beta_{1}$-AR also found that a cardiomyopathy developed (24).

We speculated that chronic activation of $\beta$-adrenergic signaling is responsible for the development of cardiomyopathy in the older $G_{s \alpha}$ mice. To prove this point, it was necessary to determine whether chronic blockade of the $\beta$-adrenergic signaling pathway could prevent the development of the cardiomyopathy, which was the goal of the current investigation. Because of the chronicity of the disease process, the effects of prolonged $\beta$-AR blockade (6-7 months' duration) was studied.

Propranolol treatment prevented the cardiac dilation and decline in LV function characteristic of cardiomyopathy. Most strikingly, the premature mortality was abolished. The myocyte hypertrophy and fibrosis were essentially prevented, as they were no longer different from the values observed in the 9month-old control animals, i.e., at a time before treatment was initiated. These data suggest that chronic $\beta$ AR blockade can arrest the development of cardiomyopathy. The beneficial effects of $\beta$-AR blockade on cardiac fibrosis in response to hypertension $(25,26)$, norepinephrine $(27)$ and myocardial infarction (28) have also been shown. Even more interest- 
ing, myocyte apoptosis, already present at 9 months of age in the TG mice, was also essentially eliminated by propranolol treatment. It has been demonstrated that apoptosis occurs in the hearts of patients with ischemic and dilated cardiomyopathy and end-stage heart failure $(29,30)$, and in animal models of heart failure $(19,31-33)$. It has also been shown that $\beta$-AR stimulation increases the percentage of apoptotic cardiocytes $(13,34,35)$. The unique finding of the current investigation is that the apoptotic process can be eliminated, once initiated, and this may have important implications for the pathogenesis of cardiomyopathy. This differs from the data on myocyte hypertrophy and collagen accumulation. These processes were attenuated, but not reversed, by the propranolol treatment. It is possible that with treatment initiated earlier, no hypertrophy or collagen accumulation would be observed.

It is generally thought that apoptotic cell death results in little or no inflammatory response and scar formation $(36,37)$. The observation of apoptosis, along with necrosis and replacement fibrosis in the older mouse with overexpressed $\mathrm{G}_{\mathrm{s} \alpha}(13)$, suggests that 2 independent pathways of cell death occur. Conversely, in the propranolol-treated $\mathrm{G}_{\mathrm{s} \alpha}$ mice, both pathways are inhibited together. Also, there was a difference in the effects of propranolol treatment on apoptosis versus hypertrophy and collagen accumulation: apoptosis already present at 9 months, at levels approaching those expected at 16 months, was essentially eliminated with propranolol treatment, but the hypertrophic and collagen accumulation processes already present at 9 months, but at levels for less than those expected at 16 months, were arrested from further development, but not eliminated.

It is also generally assumed that myocyte apoptosis is a relatively rapid phenomenon. If this is correct, then over a prolonged period, the $0.1-0.2 \%$ apoptosis observed in this study might lead to a decrease in heart size. Indeed, this may explain why the increase in $\mathrm{LV} /$ body weight is so modest in the face of major increases in myocyte cell size in this model of cardiomyopathy. However, it is also possible that the apoptotic process is relatively prolonged in the heart, which could diminish its impact over time. Finally, it is possible that apoptosis is accompanied by myocyte hyperplasia, which would result in little net gain or net loss of cardiac mass.

In patients with heart failure, selective and nonselective $\beta$-AR blockade exert beneficial effects on cardiac function and decrease cardiac mortality. In the current study, nonselective $\beta$-AR blockade was used. Therefore, we could not ascertain whether the beneficial effects were mediated by blocking either $\beta_{1}$-AR or $\beta_{2}$-AR.

In conclusion, the results of the current investigations support the concept that patients with LV dysfunction and chronically enhanced $\beta$-adrenergic signaling would be best treated by early initiation of $\beta$-AR blockade therapy. In this regard, data now exist from several large human heart failure studies demonstrating that $\beta$-AR blockade both decreases mortality and improves the heart failure syndrome (38-41).

1. Hittinger, L., et al. 1995. Hemodynamic mechanisms responsible for reduced subendocardial coronary reserve in dogs with severe left ventricular hypertrophy. Circulation. 92:978-986.

2. Koch, W.J., et al. 1995. Cardiac function in mice overexpressing the $\beta$ adrenergic receptor kinase or a $\beta$ ARK inhibitor. Science. 268:1350-1353.

3. Milano, C.A., et al. 1994. Enhanced myocardial function in transgenic mice overexpressing the $\beta_{2}$-adrenergic receptor. Science. 264:582-586.

4. Rohrer, D.K., et al. 1996. Targeted disruption of the mouse $\beta 1$-adrenergic receptor gene: developmental and cardiovascular effects. Proc. Natl. Acad. Sci. USA. 93:7375-7380.

5. Wakasaki, H., et al. 1997. Targeted overexpression of protein kinase C $\beta 2$ isoform in myocardium causes cardiomyopathy. Proc. Natl. Acad. Sci. USA. 94:9320-9325.

6. Hein, L., et al. 1997. Overexpression of angiotensin $\mathrm{AT}_{1}$ receptor transgene in the mouse myocardium produces a lethal phenotype associated with myocyte hyperplasia and heart block. Proc. Natl. Acad. Sci. USA. 94:6391-6396.

7. Conner, D.A., et al. 1997. $\beta$-Arrestin 1 knockout mice appear normal but demonstrate altered cardiac responses to $\beta$-adrenergic stimulation. Circ. Res. 81:1021-1026.

8. Gaudin, C., et al. 1995. Overexpression of $\mathrm{G}_{\mathrm{s} \alpha}$ protein in the hearts of transgenic mice. J. Clin. Invest. 95:1676-1683.

9. Iwase, M., et al. 1996. Adverse effects of chronic endogenous sympathetic drive induced by cardiac $\mathrm{G}_{\mathrm{s} \alpha}$ overexpression. Circ. Res. 78:517-524.

10. Uechi, M., et al. 1998. Depressed heart rate variability and arterial baroreflex in conscious transgenic mice with overexpression of cardiac $G_{s \alpha}$. Circ. Res. 82:416-423.

11. Kim, S.J., et al. 1999. Differential regulation of inotropy and lusitropy in overexpressed Gs $\alpha$ myocytes through cAMP and $\mathrm{Ca}^{2+}$ channel pathways. J. Clin. Invest. 103:1089-1097.

12. Vatner, D.E., et al. 1998. Overexpression of myocardial Gs $\alpha$ prevents full expression of catecholamine desensitization despite increased $\beta$-adrenergic receptor kinase. J. Clin. Invest. 101:1916-1922.

13. Geng, Y.J., et al. 1999. Apoptosis of cardiac myocytes in Gs $\alpha$ transgenic mice. Circ. Res. 84:34-42.

14. Iwase, M., et al. 1997. Cardiomyopathy induced by cardiac $\mathrm{G}_{\mathrm{s}} \alpha$ overexpression. Am. J. Physiol. 272:H585-H589.

15. National Institutes of Health, Office of Science and Health Reports. 1996. Guide for care and use of laboratory animals. Publication no. 83-23. Office of Science and Health Reports, Department of Health and Human Services. Bethesda, MD.

16. Fentzke, R.C., Korcarz, C.E., Lang, R.M., Lin, H., and Leiden, J.M. 1998. Dilated cardiomyopathy in transgenic mice expressing a dominant-negative CREB transcription factor in the heart. J. Clin. Invest. 101:2415-2426.

17. Arber, S., et al. 1997. MLP-deficient mice exhibit a disruption of cardiac cytoarchitectural organization, dilated cardiomyopathy, and heart failure. Cell. 88:393-403.

18. Sato, Y., et al. 1998. Cardiac-specific overexpression of mouse cardiac calsequestrin is associated with depressed cardiovascular function and hypertrophy in transgenic mice. J. Biol. Chem. 273:28470-28477.

19. Kubota, T, et al. 1997. Dilated cardiomyopathy in transgenic mice with cardiac-specific overexpression of tumor necrosis factor- $\alpha$. Circ. Res. 81:627-635.

20. Franco, F., et al. 1999. Magnetic resonance imaging and invasive evaluation of development of heart failure in transgenic mice with myocardial expression of tumor necrosis factor- $\alpha$. Circulation. 99:448-454.

21. Edwards, J.G., et al. 1996. Cardiomyopathy in transgenic myf5 mice. Circ. Res. 78:379-387.

22. Sussman, M.A., et al. 1998. Myofibril degeneration caused by tropomodulin overexpression leads to dilated cardiomyopathy in juvenile mice. J. Clin. Invest. 101:51-61.

23. Colbert, M.C., et al. 1997. Cardiac compartment-specific overexpression of a modified retinoic acid receptor produces dilated cardiomyopathy and congestive heart failure in transgenic mice. J. Clin. Invest. 100:1958-1968.

24. Engelhardt, S., Hein, L., Wiesmann, F., and Lohse, M.J. 1999. Progressive hypertrophy and heart failure in $\beta_{1}$-adrenergic receptor transgenic mice. Proc. Natl. Acad. Sci. USA. 96:7059-7064.

25. Ieki, K., et al. 1989. Effect of long-term treatment with $\beta$-blocker on cardiac hypertrophy in SHR. J. Mol. Cell. Cardiol. 21(Suppl. 5):113-119.

26. Barone, F.C., Campbell, W.G., Jr., Nelson, A.H., and Feuerstein, G.Z. 1998. Carvedilol prevents severe hypertensive cardiomyopathy and remodeling. J. Hypertens. 16:871-884.

27. Rosenbaum, J.S., Ginsburg, R., Billingham, M.E., and Hoffman, B.B. 1987. Effects of adrenergic receptor antagonists on cardiac morphological and functional alterations in rats harboring pheochromocytoma. J. Pharmacol. Exp. Ther. 241:354-360. 
28. Latini, R., et al. 1998. Comparative efficacy of a $\mathrm{DA}_{2} / \alpha_{2}$ agonist and a $\beta$ blocker in reducing adrenergic drive and cardiac fibrosis in an experimental model of left ventricular dysfunction after coronary artery occlusion. J. Cardiovasc. Pharmacol. 31:601-608.

29. Narula, J., et al. 1996. Apoptosis in myocytes in end-stage heart failure. N. Engl. J. Med. 335:1182-1189.

30. Olivetti, G., et al. 1997. Apoptosis in the failing human heart. N. Engl. J. Med. 336:1131-1141.

31. Liu, Y., et al. 1995. Myocyte nuclear mitotic division and programmed myocyte cell death characterize the cardiac myopathy induced by rapid ventricular pacing in dogs. Lab. Invest. 73:771-787.

32. Teiger, E., et al. 1996. Apoptosis in pressure overload-induced heart hypertrophy in the rat. J. Clin. Invest. 97:2891-2897.

33. Cheng, W., et al. 1995. Stretch-induced programmed myocyte cell death. J. Clin. Invest. 96:2247-2259.

34. Shizukuda, Y., et al. 1998. $\beta$-Adrenergic stimulation causes cardiocyte apoptosis: influence of tachycardia and hypertrophy. Am. J. Physiol. 275:H961-H968.
35. Communal, C., Singh, K., Pimentel, D.R., and Colucci, W.S. 1998. Norepinephrine stimulates apoptosis in adult rat ventricular myocytes by activation of the $\beta$-adrenergic pathway. Circulation. 98:1329-1334.

36. Anversa, P. 1998. Myocyte apoptosis and heart failure. Eur. Heart J. 19:359-360

37. Yeh, E.T. 1997. Life and death in the cardiovascular system. Circulation. 95:782-786.

38. CIBIS Investigators and Committees. 1994. A randomized trial of $\beta$ blockade in heart failure. The Cardiac Insufficiency Bisoprolol Study (CIBIS). Circulation. 90:1765-1773.

39. Lechat, $P$., et al. 1998. Clinical effects of $\beta$-adrenergic blockade in chronic heart failure: a meta-analysis of double-blind, placebo-controlled, randomized trials. Circulation. 98:1184-1191.

40. Packer, M., et al. 1996. The effect of carvedilol on morbidity and mortality in patients with chronic heart failure. N. Engl. J. Med. 334:1349-1355.

41. CIBIS Investigators and Committees. 1999. The Cardiac Insufficiency Bisoprolol Study II (CIBIS-II): a randomised trial. Lancet. 353:9-13. 